conducted annually by a self-reported questionnaire since 2001. Inclusion criteria are a diagnosis of SLE and returning the completed paper questionnaire. Amongst others medication, comorbidities, health-related quality of life (HRQoL, Short-Form-12), and disease activity (Systemic Lupus Activity Measure) are surveyed. In the year 2015 we additionally inquired about participation using the "Index zur Messung von Einschränkungen der Teilhabe" (IMET; Index for measuring limitations of participation) that was developed on the basis of the International Clas sification of Functioning, Disability and Health (ICF), as well as depression (Centre for Epidemiologic Studies Depression Scale), and pain coping (Pain Related Self statements scale).

A multiple linear regression was run to predict overall impairment and impairment in the individual subdomains (dependent variables). Age, disease duration, number of comorbidities, pain, disease activity, catastrophizing, coping, depression, physical functioning, and physical and mental HRQoL were entered into our model as candidates for the independent variables. Variable selection was accomplished by a stepwise approach based on Akaike information criterion (AIC).

Results: The questionnaire was completed by 579 patients (response rate $89.2 \%)$. Only $48(8.3 \%)$ reported no impairment of participation by their disease. Most limitations were reported in the domains 'stress and extraordinary strains' (56.3\% reported moderate to high impairment) and 'sex life' (48.7\%), whereas 'common activities of daily life' (21.1\%) and 'close personal relationships' $(26.5 \%)$ seemed to be limited less frequently.

Depression, physical functioning, and physical and mental HRQoL predicted overall participation (F-test, $p<0.0001$ ).

Depression, physical functioning, and physical HRQoL predicted also most of the participation subdomains whereas age, disease duration, no. of comorbidities, disease activity, pain, and pain coping behaviour impacted only individual subdomains.

Conclusions: Limitations of participation are common in SLE patients and affect different areas of life. In order to improve participation, it is of great importance to maintain, respectively improve physical and mental quality of life, physical functioning, and depression. The direction of causality cannot be proved beyond reasonable doubt in this cross-sectional analysis. Additional longitudinal studies are necessary.

Acknowledgements: The LuLa-study is supported by unrestricted grants from GlaxoSmithKline and UCB Pharma

Disclosure of Interest: $G$. Chehab Grant/research support from: GlaxoSmithKline and UCB Pharma for performing the LuLa-study., J. Richter Grant/research support from: GlaxoSmithKline and UCB Pharma for performing the LuLa-study., R. Fischer-Betz Grant/research support from: GlaxoSmithKline and UCB Pharma for performing the LuLa-study., R. Brinks: None declared, B. Winkler-Rohlfing: None declared, M. Schneider Grant/research support from: GlaxoSmithKline and UCB Pharma for performing the LuLa-study.

DOI: 10.1136/annrheumdis-2018-eular.3229

\section{SAT0435 \\ PLASMA PTX3 LEVELS CORRELATE WITH SYSTEMIC LUPUS ERYTHEMATOSUS ACTIVITY AND ARE INFLUENCED BY CORTICOSTEROIDS}

G.A. Ramirez ${ }^{1,2}$, E.P. Bozzolo ${ }^{2}$, V. Canti $^{2}$, B. Bottazzi ${ }^{3}$, A. Mantovani ${ }^{3,4}$ L. Dagna ${ }^{1,2}$, P. Rovere-Querini ${ }^{1,2}$, A.A. Manfredi ${ }^{1,2}$. ${ }^{1}$ Università Vita-Salute San Raffaele; ${ }^{2}$ Unit of Immunology, Rheumatology Allergy and Rare Diseases, IRCCS San Raffaele Hospital, Milan; ${ }^{3}$ IRCCS Humanitas Research Hospital; ${ }^{4}$ Humanitas University, Rozzano (MI), Italy

Background: Systemic lupus erythematosus (SLE) is an autoimmune disease characterised by a variable involvement of multiple organs and tissues. Acute and/or chronic vascular inflammation is not uncommon in patients with SLE and can significantly affect patient quality of life and survival. Specific markers of vascular inflammation in SLE are lacking. Pentraxin-3 (PTX3) is an evolutionarily conserved pattern recognition receptor expressed by multiple cell lines and is growingly recognised as a marker of the vessel response to injury. Glucocorticoids are known inducers of PTX3 in most tissues. The role of PTX3 as a biomarker in SLE is discussed.

Objectives: To assess the potential informative role of PTX3 as a biomarker in patients with SLE with and without current or previous vasculitic manifestations and with active or quiescent disease.

Methods: We enrolled 55 adult patients with SLE for a total of 60 samples. Samples were classified as taken from patients with active disease (SLE disease activity index, SLEDAI, $\geq 4$ ) with or without active vasculitis and from patients with quiescent disease (SLEDAI <4). Further stratification was performed according to a history of lupus vasculitis. Five patients were bled twice under different conditions. Plasma PTX3 was measured by ELISA. Non-parametric tests were employed to compare PTX3 levels among groups.

Results: PTX3 plasma levels were slightly but not significantly more elevated in patients with active vasculitis. PTX3 levels correlated with SLEDAI in the whole set of patients $(p=0.007)$ and in those who were off corticosteroids $(p<0.001)$, but not in patients receiving prednisone. PTX3 levels correlated with the dose of prednisone $(p<0.001)$. Patients with $>1$ moderately-to-highly active $(A, B)$ British Isles Lupus Assessment Group (BILAG) domain had significantly higher PTX3 levels than those with more limited disease activity extent $(p=0.041)$. PTX3 also correlated with a 0.0-3.0 physician global assessment scale (PGA), with patientreported visual analogue scale, and inversely with $C 4$ levels $(p=0.004, p=0.013$, $\mathrm{p}=0.001$ respectively). There was no significant correlation with age or disease duration nor with C-reactive protein (CRP). Similar to PTX3, CRP was higher in patients with $>1$ A/B BILAG domain $(p=0.004)$, but did not correlate with SLEDA or prednisone dose. Repeated samples showed a high intra-individual variability for PTX3, which unpredictably correlated with disease activity and prednisone dosage.

Conclusions: Our data suggest that PTX3 is a marker of active disease extent rather than vascular inflammation in SLE and it shares this behaviour with CRP, another member of the pentraxin family. Nonetheless, PTX3 also specifically cor relate with monoparametric indexes of activity such as SLEDAI. A high intra-individual variability and the effect of corticosteroids constitute potential limitations to future diagnostic applications of PTX3 in SLE.

\section{REFERENCES:}

[1] Erreni M, et al. Immunol Rev 2017

[2] Skare T, et al. Joint Bone Spine 2015.

[3] Assandri R, et al. Autoimmune Diseases 2015

Disclosure of Interest: None declared

DOI: 10.1136/annrheumdis-2018-eular.7317

\section{SAT0436 TRANSJUGULAR RENAL BIOPSY: A SAFE AND EFFECTIVE WAY TO PERFORM RENAL BIOPSY IN SYSTEMIC LUPUS ERYTHEMATOSUS AND ANTIPHOSPHOLIPID ANTIBODY SYNDROME PATIENTS TREATED WITH ANTI-THROMBOTIC DRUGS - A MONOCENTRIC EXPERIENCE OF 256 PROCEDURES}

H. Nielly ${ }^{1}$, A. Mathian ${ }^{1,2,3}$, M. Cazenave ${ }^{1}$, H. Izzedine ${ }^{4}$, J. Haroche ${ }^{1,2,3}$, F. Cohen Aubart $^{1,2,3}$, P. Rouvier ${ }^{5}$, I. Brocheriou ${ }^{3,5}$, P. Cluzel ${ }^{3,6}$, Z. Amoura $^{1,2,3}{ }^{1}{ }^{1}$ French national reference center for systemic lupus erythematosus and antiphospholipid antibody syndrome, service de Médecine interne 2, Institut E3M, Assistance Publique-Hôpitaux de Paris (AP-HP), Groupement Hospitalier Pitié-Salpêtrière (GHPS); ${ }^{2}$ U1135, Centre d'Immunologie et des Maladies Infectieuses (CIMI-Paris), INSERM; ${ }^{3}$ Université Pierre et Marie Curie, Université Pierre et Marie Curie; ${ }^{4}$ service de néphrologie, Assistance Publique-Hôpitaux de Paris (AP-HP), Groupement Hospitalier Pitié-Salpêtrière (GHPS); ${ }^{5}$ Service d'Anatomie et Cytologie Pathologiques, Assistance Publique-Hôpitaux de Paris (AP-HP), Groupement Hospitalier Pitié-Salpétrière; ${ }^{6}$ Département d'imagerie cardiovasculaire et de radiologie interventionnelle, Assistance Publique-Hôpitaux de Paris (AP-HP), Groupement Hospitalier Pitié-Salpêtrière (GHPS), Paris, France

Background: Renal biopsy is the cornerstone of Lupus nephritis (LN) management. However, transcutaneous renal biopsy (TCRB) is hampered by the antithrombotic treatment frequently prescribed in Systemic Lupus Erythematosus (SLE) and Antiphospholipid Antibody Syndrome (APS). Transjugular renal biopsy (TJRB) offers an attractive alternative for patients at increased risk of bleeding. Objectives: The primary objective of the study was to describe the safety and the diagnostic performance of TJRB in SLE and APS.

Methods: A retrospective review of SLE and/or APS patients who consecutively underwent a renal biopsy in our department between January 2004 and October 2016 was performed. Biopsies were divided into four groups: TCRB, TJRB with aspirin treatment (aspirin TJRB), TJRB with anticoagulant treatment (anticoagulant TJRB), and TJRB without anti-thrombotic drug (no-antithrombotic TJRB). Major complications were defined as decease, haemostasis nephrectomy, renal artery embolization, blood transfusion, sepsis and vascular thrombosis. Minor complications were defined as gross haematuria, renal hematoma and arteriovenous fistula.

Results: Fifty-four TCRB and 256 TJRB were analysed - 69 aspirin TJRB, 68 anticoagulant TJRB and 119 no-antithrombotic TJRB. Major complications rate was $1.9 \%$ for TCRB and $2.0 \%$ for TJRB $(p=1)$. One patient in the TJRB group suffering from catastrophic antiphospholipid syndrome (CAPS) died suddenly 6 weeks after the biopsy. No patient died of bleeding complication. One patient in the anticoagulant TJRB group required a renal artery embolization and blood transfusion. Four other patients required blood transfusion (1 in the TCRB group 1 in the aspirin TJRB group and 2 in the anticoagulant TJRB group). Minor complications rate was $1.9 \%$ for TCRB and $7.8 \%$ for TJRB $(p=0.2)$.

Among the 256 TJRB, the rate of complication (major or minor) was higher for patients with glomerular filtration rate CKD-EPI $<30 \mathrm{~mL} / \mathrm{min}(6 / 24$ [25\%]) compared to patients with GFR $>30 \mathrm{~mL} / \mathrm{min}(16 / 232$ [7\%], $\mathrm{p}<0.01$ using the Khi-2 test). Age over 40, blood pressure $>140 / 90 \mathrm{mmHg}$, APS or positive 